\title{
Uncertainty and Central Bank Transparency: A Non-Bayesian Approach
}

\author{
Daniel Laskar* \\ Paris School of Economics, CNRS and CEPREMAP, France
}

September 27, 2010

\begin{abstract}
We underline that some results obtained in the literature on central bank transparency may be quite different when we take a non-Bayesian approach to uncertainty, where "ambiguity" is taken into account. We consider some specific argument of the literature (obtained under a Bayesian approach), which implies that political uncertainty can be beneficial and central bank transparency harmful. We show that when ambiguity is large enough, these results do not hold anymore: political uncertainty is always harmful and central bank transparency always beneficial. Furthermore, as soon as we depart from the Bayesian case, Knightian uncertainty is always harmful.
\end{abstract}

JEL Classification: E58; E52

Keywords: central bank transparency; political uncertainty; Knightian uncertainty; ambiguity; non-Bayesian approach

*Address: CEPREMAP, 142 rue du Chevaleret, 75013 Paris, France. Ph: 33 (0)1 40 7784 08. Fax: 33 (0)1 442438 57. E-mail: laskar@pse.ens.fr 


\section{Introduction}

In the last two decades, there has been a widespread move of central banks toward more transparency. Such a move has in part been due to the trend toward more central bank (CB) independence. Being more independent, CBs had to be more accountable and, for that, had to be more transparent about the way they conducted their monetary policies. But there are also economic reasons for CBs to be more transparent. By providing additional information on the underlying factors affecting monetary policy (objectives of the $\mathrm{CB}$, economic data and forecasts made by the $\mathrm{CB}$, procedures involved in the decision process,...) transparency can help the private sector to make better decisions. As a consequence, there has been a large development of both the theoretical and the empirical literature on the economic effects and possible usefulness of increased CB transparency.

Theoretical studies have mixed conclusions. Although some results are favorable to transparency, there are also several types of arguments pointing to the possible deficiencies of increased transparency. Thus, the results obtained in the literature depend on the model considered and on the specific assumptions made ${ }^{1}$.

In this paper, we will develop the argument that the results obtained in the literature may also depend on the approach to uncertainty which is taken. The theoretical literature on CB transparency usually adopts a Bayesian expected utility criterion ${ }^{2}$. However, Knight (1921) already argued that a distinction should be made between a situation of "risk", where there is some known objective probability distribution, and a situation of "(Knightian) uncertainty", where this is not the case. Furthermore, some insufficiencies of the Bayesian approach (where uncertainty is represented by a subjective probability distribution) in describing behavior have been pointed out. Thus,

\footnotetext{
${ }^{1}$ From a rather general point of view, if we have a situation where there are some inefficiencies in the economy, then this can make a lack of transparency beneficial, because a less informed private sector may help to reduce these inefficiencies. Depending on the model or on the specific assumptions made, this may or may not occur.

Concerning empirical results, although there are some differences in the results obtained in the various specific studies, broadly speaking, the overall picture which arises from the empirical literature seems to be that CB transparency does have an impact on the private sector's behavior. Financial markets and some macroeconomic variables like expected inflation appear to be favorably affected.

For some recent surveys of the theoretical and of the empirical literature on CB transparency, see, for example, Blinder and al. (2008) and van der Cruisen and Eijffinger (2007).

${ }^{2}$ An exception is Schipper and Winschel (2004). They introduce Knightian uncertainty in an ambiguous game framework. The similarities and differences between their analysis and the analysis of the present paper are mentioned in Section 3 below.
} 
the Ellsberg paradox (Ellsberg (1961)) has underlined the existence of some "aversion to ambiguity". Therefore, in the last two or three decades, new approaches to uncertainty, where such an aversion to ambiguity is introduced, and which encompass the Bayesian approach as a special case, have been developed ${ }^{3}$.

Here, we will use such a non-Bayesian approach to uncertainty. We will consider some rather simple specific argument against central bank transparency which is found in the literature. Then, we will show that the results can be overturned when we depart from the Bayesian case. Instead of being a case against transparency, the argument can become a case for transparency.

The specific argument of the literature that we will consider, underlines that increased uncertainty on the CB's preferences may be beneficial through its favorable effect on the level of the nominal wage (Grüner (2002) and Sorensen (1991)). The argument relies on the analysis of a game between a monopoly labor union and a CB. The labor union sets the nominal wage before the $\mathrm{CB}$ chooses its monetary policy. As the weight the $\mathrm{CB}$ attaches to its inflation objective relatively to its unemployment objective is not known to the labor union, this creates some uncertainty on how the CB reacts to the nominal wage. It is then shown that more uncertainty decreases the level of the nominal wage chosen by the labor union. As a consequence, this may improve macroeconomic performance defined in terms of unemployment and inflation, and therefore be beneficial ${ }^{4}$.

In this analysis of the literature, uncertainty is represented by a probability distribution, and a standard expected utility criterion is used. More uncertainty is associated with a greater variance of the probability distribution. In Sorensen (1991), the source of the uncertainty is assumed to be some political factors, and the implication of the result is that political uncertainty, which creates variability in the weight between the CB's objectives, may be beneficial. In Grüner (2002), this result is rather interpreted as an argument against too much transparency, where more transparency is assumed to imply a lower variance of the probability distribution.

\footnotetext{
${ }^{3}$ Two classical references are Gilboa and Schmeidler (1989) and Schmeidler (1989). In Gilboa and Schmeidler (1989), the decision maker has a set of probability distributions, instead of a unique probability distribution, and uses a maxmin criterion over this set of distributions. In Schmeidler (1989), non additive probabilities ("capacities") are used.

In these non-Bayesian frameworks, the presence of some aversion to ambiguity leads the decision maker to give more weight to the bad outcomes implied by each decision.

A survey of some economic applications can be found in Mukerji and Tallon (2004).

${ }^{4}$ This argument has been developed by Sorensen (1991). Grüner (2002) gave an interpretation in terms of CB transparency, and also showed that reduced uncertainty on the CB's preferences (which may be due to more CB transparency) may actually increase inflation uncertainty.
} 
As we have indicated, in the present paper, we will revaluate this argument by using a non-Bayesian approach, where the decision maker has some aversion to ambiguity. Such a framework will allow us to introduce Knightian uncertainty into the analysis. As we want to compare situations which are more or less uncertain in some sense, we need to consider the effects of changes in the information available to the decision maker. Therefore, we will consider a non-Bayesian approach which makes explicit the information available to the decision maker ${ }^{5}$. We will use the approach of Gajdos et al. (2004), which introduces some aversion to ambiguity under the form of an "aversion to the imprecision of information". Under this approach, the information available to the decision maker consists in two things: a "central probability distribution"; and a set of possible probability distributions around this central probability distribution, which represents Knightian uncertainty around this central distribution.

We will assume that political factors create some underlying fluctuations in the CB's preferences which follow some given probability distribution. This probability distribution is only imperfectly known to the private sector. In the information available to the private sector, the central probability distribution is an estimate of this probability distribution obtained under some central prior, while Knightian uncertainty around this central distribution represents the sensitivity of the estimates to other priors. Increased CB transparency reduces both the variance of the central distribution and the amount of Knightian uncertainty.

The presence of Knightian uncertainty and of some aversion to ambiguity will lead to results which are different, and often opposite to those obtained in the literature under a Bayesian approach. First, we will find that an increase in the variance of the central distribution, which may be due to more variable political factors or to less CB transparency, does not affect the nominal wage when we are far enough from the Bayesian case. Consequently, in this case, this greater variance can never be beneficial and is in general harmful. Second, we will find that the nominal wage is never lowered but, on the contrary, is often raised by an increase in Knightian uncertainty. As a consequence, more Knightian uncertainty is, in general, always harmful. These results tend to make more political uncertainty harmful, and more CB transparency beneficial. This is opposite to what was underlined in the literature under the Bayesian approach.

Our framework of analysis is presented in Section 2. In Section 3, the

${ }^{5}$ This is not the case for the two classical approaches previously mentioned. In Gilboa and Schmeidler (1989), or in Schmeidler (1989), the information available to the decision maker is not made explicit. 
equilibrium nominal wage is determined. The effects on the nominal wage of changes in the variance of the central distribution, or of the amount of Knightian uncertainty, is considered. The welfare effects are examined in Section 4. Section 5 concludes.

\section{Framework of analysis}

\subsection{Model}

As we only want to change the way uncertainty is treated, we will take the same model as in Grüner $(2002)^{6}$. The model is static. There is a unique labor union (LU) in the labor market and we consider a two-stage game between the LU and the central bank (CB). The LU fixes the nominal wage at the beginning of the period, and the $\mathrm{CB}$ subsequently determines the inflation rate. The equations are the following:

$$
\begin{array}{rlrl}
U^{C B} & =-\psi \pi^{2}-u^{2} \quad ; \quad & \psi>0 \\
U^{L U} & =w-\pi-\frac{A}{2} u^{2} \quad ; \quad & A>0 \\
u & =a(w-\pi) \quad ; \quad a>0
\end{array}
$$

$U^{C B}$ and $U^{L U}$ represent the utility functions of the $\mathrm{CB}$ and of the $\mathrm{LU}$, respectively. The $\mathrm{CB}$ tries to stabilize both inflation $\pi$ and unemployment $u$ around some desired levels, with a relative weight given by $\psi$ (the desired levels for inflation and employment are normalized to zero). The LU wants to stabilize unemployment around the same level as the $\mathrm{CB}$ (with a relative weight given by $A$ ), and to increase the (log) real wage $w-\pi$, where $w$ represents the $(\log )$ nominal wage (we use the normalization $p_{-1}=0$, where $p_{-1}$ is the (log) price level of the previous period, which implies $\pi=p$ ). After the nominal wage has been fixed, unemployment is determined by labor demand, according to equation (3), where unemployment is an increasing function of the real wage ${ }^{7},{ }^{8}$.

\footnotetext{
${ }^{6}$ This model is itself taken from the existing literature analyzing the interaction of labor unions and the $\mathrm{CB}$, and its implications for monetary policy and the macroeconomic equilibrium (Cukierman and Lippi (1999)).

${ }^{7}$ As in Grüner (2002), there is no shock in equation (3). Note that by a suitable normalization of the nominal wage and price variables, the constant term in this equation can always be taken equal to zero.

${ }^{8}$ We could as well assume that, as in Sorensen (1991), the LU has only a real wage objective. For, using (2) and (3), we obtain $U^{L U}=-\frac{A a^{2}}{2}\left(w-\pi-\omega^{*}\right)^{2}+\frac{1}{2 A a^{2}}$, where
} 
The equilibrium of the game is solved backward. In the second stage, once $w$ has been fixed by the LU, the $\mathrm{CB}$ chooses $\pi$ which maximizes $U^{C B}$ under the constraint (3). This leads to the reaction function $\pi=b w$, where $b=\frac{a^{2}}{a^{2}+\psi}$. Using the notation $\gamma \equiv 1-b$, we get

$$
\gamma=\frac{\psi}{a^{2}+\psi}
$$

We have $0<\gamma<1$. The CB's reaction function can then be written:

$$
w-\pi=\gamma w
$$

In the first stage, the LU, when choosing $w$, takes into account the reaction function (5) of the CB. Using (2), (3) and (5) we get

$$
U^{L U}=\gamma w-\frac{A a^{2}}{2} \gamma^{2} w^{2}
$$

It is assumed that the LU does not know the CB's preferences. As $\psi$ is unknown, $\gamma$ is also unknown. We now have to be more explicit on how we model this uncertainty on $\gamma^{9}$.

\subsection{Uncertainty}

\subsubsection{Available information}

As in Sorensen (1991), varying political factors make the CB's preferences fluctuate. Therefore, we will assume that parameter $\gamma$ is a random variable which follows some probability distribution $P^{*}$. Let $\gamma_{0}$ and $\sigma^{* 2}$ be the mean and variance of $P^{*}$, respectively.

Suppose that $P^{*}$ depends on some underlying parameters that are not perfectly known. The LU tries to estimate these underlying parameters by using some available data. For that, the LU first does some Bayesian statistical analysis where data are combined with some prior that the LU may have

$\overline{\omega^{*}}=\frac{1}{A a^{2}}>0$ would be the LU's real wage desired level.

Note that, using (3), this implies $U^{L U}=-\frac{A}{2}\left(u-a \omega^{*}\right)^{2}+\frac{1}{2 A a^{2}}$. Therefore, the LU could also as well be considered as having an implicit unemployment desired level $u^{* L U}=$ $a \omega^{*}=\frac{1}{A a}>0$, which is higher than the CB's unemployment desired level (which is equal to zero).

${ }^{9}$ As in Grüner (2002), we focus on the random variable $\gamma$ (or equivalently $b$ ). However, as underlined by Dai and Sidiropoulos (2008), the results can be modified if we take the variance of $\psi$, rather than the variance of $\gamma$, as the parameter which decreases when transparency increases. Theses authors show that this can give a role to the degree of conservativeness of central bankers. 
on the underlying parameters. This will lead to some posterior probability distribution on these parameters. Then, this posterior probability distribution on the underlying parameters of $P^{*}$ can be used to give a probability distribution on $\gamma$. Let us call $C$ this "central probability distribution" on $\gamma$. In order to simplify the analysis and to get rid of any role that a bias in the mean of $C$ may play in our analysis, we assume that $C$ has the same mean $\gamma_{0}$ as $P^{*}$. Let $\sigma_{\gamma}^{2}$ be the variance of $C$.

The distribution $C$ depends on the prior held on the underlying parameters of $P^{*}$. Other priors would have given different distributions. Therefore, the LU also does some robustness analysis, by taking some set of priors around the prior used in order to get $C$. This leads to a set $\mathcal{P}$ of probability distributions around $C$. This lack of confidence put on the central distribution $C$ may be viewed as "Knightian uncertainty". We will assume that this set $\mathcal{P}$ is given by ${ }^{10}$

$$
\mathcal{P}=\{(1-\delta) C+\delta P: P \in \mathcal{R}(S)\}
$$

where $S$ is some subset of $[0,1]$ which is known to contain the support of $P^{*}$ (and therefore the support of $C$ is also assumed to be in $S$ ). $\mathcal{R}(S)$ is the set of all probability distributions having support contained in $S$. This means that any possible distribution can be written as a weighted average of the central distribution $C$ and of a distribution with support contained in $S$. (Therefore, the support of any possible distribution is also in $S$ ).

Parameter $1-\delta$, where we have $0 \leq \delta \leq 1$, measures the degree of confidence put on the central distribution $C$. A larger value of $\delta$ implies a greater imprecision of information around $C$, and therefore increases the amount of Knightian uncertainty. In the limit case $\delta=0$, we have $\mathcal{P}=\{C\}$, and Knightian uncertainty disappears. In the other limit case $\delta=1$, we have $\mathcal{P}=\mathcal{R}(S)$. Then, there is complete ignorance (except for the fact that all possible distributions have support in $S$ ).

\footnotetext{
${ }^{10}$ Such a set of probability distributions, defined by "contamination" from a given probability distribution, has been considered in the literature on robust Bayesian analysis (see Berger and Berliner (1986)). But, in this literature, this concerned the class of "priors" on the underlying parameters, before data had been used. Here, it concerns the distributions on $\gamma$ which result from the "posterior" distributions on the underlying parameters, after data have been used.

Such a class of probability distributions, defined by "contamination", has also been used by Epstein and Wang (1994). However, in Epstein and Wang (1994) it does not represent the available information, but the probability set used in a Gilboa and Schmeidler (1989) framework, where the information available is not made explicit. It therefore represents a mix of the uncertainty on the information available, on the one hand, and of the aversion to uncertainty, on the other hand. In our framework, however, it only concerns the information available to the decision maker, while some kind of aversion to uncertainty will be separately considered (and is represented by parameter $\theta$ below in the text).
} 
In order to simplify the analysis, we will further assume that $S$ is a symmetric interval around $\gamma_{0}$. We take $S=\left[\gamma_{0}-\mu, \gamma_{0}+\mu\right]$, where $\mu>0$ is a parameter which represents the length of that interval. As we have $0<\gamma<1$, parameter $\mu$ has to satisfy the inequalities $\mu<\gamma_{0}$ and $\mu<1-\gamma_{0}$.

Thus, the information available to the $\mathrm{LU}$ is given by $\{C, \mathcal{P}\}$.

\subsubsection{Criterion}

We will use the approach of Gajdos et al. (2004) which makes the information available to the decision maker explicit. The available information has the form we have just considered. It is given by $[C, \mathcal{P}]$, where $C$ is a "central probability distribution" around which there is some "imprecision of information", expressed by the fact that all probability distributions contained in the set $\mathcal{P}$ are considered as possible.

The axiomatic approach of Gajdos et al. (2004) ${ }^{11}$ introduces some kind of aversion to ambiguity in an axiom of "aversion to the imprecision of information". It leads to a criterion which consists in a weighted average of two terms: the expected utility under the central distribution $C$, on the one hand; and the minimum of the expected utility under the (convex hull $\operatorname{co}(\mathcal{P})$ of the) set $\mathcal{P}$ of all possible probability distributions, on the other hand (see Gajdos et al. (2004) Theorem 2 p.661) ${ }^{12}$. Therefore, the criterion which the LU maximises is given by

$$
\Omega^{L U}=(1-\theta) E_{C} U^{L U}+\theta\left[\min _{P \in \operatorname{co}(\mathcal{P})} E_{P} U^{L U}\right]
$$

where $E_{P}$ and $E_{C}$ represent the corresponding expected value operators. In this equation, parameter $\theta$, which belongs to $[0,1]$, represents the "aversion to the imprecision of information" of the LU.

\footnotetext{
${ }^{11}$ In this axiomatic approach, the preferences of the decision maker concern both the decision $f$ (or "act"), which, in the case of the LU, is equal to $w$, and the available information $[C, \mathcal{P}]$ : it is a binary relation on couples $(f,[C, \mathcal{P}])$.

${ }^{12} \mathrm{~A}$ criterion which involves a weighted average between an expected utility under a given distribution, and a minimum of expected utilities under a set of possible distributions, can also be obtained under the non-additive probabilities approach of Schmeidler (1989) (see Eichberger and Kelsey (1999)); or under the approach of Gilboa and Schmeidler (1989) when the set of distributions is defined by "contamination" (see Epstein and Wang (1994)).
} 
From (7) and (8) we get the criterion given by ${ }^{13}$

$$
\Omega^{L U}=(1-\alpha) E_{C} U^{L U}+\alpha\left[\min _{P \in \mathcal{R}(S)} E_{P} U^{L U}\right]
$$

where we have

$$
\alpha=\theta \delta
$$

This criterion is a weighted average of two terms: the expected utility under $C$, on the one hand; and the minimum of the utility for all distributions having their supports contained in $S$, on the other hand. This second term indicates that (as long as we have $\alpha \neq 0$ ), for each decision considered, the LU gives an additional weight to what happens in the worst case belonging to $\mathcal{R}(S)$, i.e. when we consider the probability distribution which gives the lowest utility level among all the probability distributions which have support in $S$. .

According to $(10)$, the weight $\alpha$ (which satisfies $0 \leq \alpha \leq 1$ ) given to this second term, is the product of two parameters: parameter $\theta$, which represents the aversion of the decision maker to the imprecision of information; and parameter $\delta$, which is an index of such an imprecision of information. Parameter $\alpha$ will represent the level of "ambiguity".

The extreme case $\alpha=0$, where ambiguity is equal to zero, corresponds to the Bayesian case where the LU maximizes expected utility under the central distribution $C$. It is obtained in two cases. The first is obtained when there is no aversion to the imprecision of information $(\theta=0)$; the second case $(\delta=0)$ occurs when there is no imprecision of information around $C$, and therefore no Knightian uncertainty.

The other extreme case $(\alpha=1)$ corresponds to the maxmin criterion under all distributions having support in $S$. It requires both $\delta=1$, i.e. complete ignorance (except knowing that the support is contained in $S$ ), and the extreme value $\theta=1$ of the aversion to the imprecision of information. In this extreme case, for each decision considered, only the worst distribution, among all the distributions having support in $S$, is taken into account.

\subsubsection{Comparative statics}

In our framework, CB transparency will affect two parameters. First, more transparency decreases the variance $\sigma_{\gamma}^{2}$ of the central distribution $C$. The

\footnotetext{
${ }^{13}$ The set $\mathcal{P}$ defined by (7) is a convex set. For, from the definition of $\mathcal{P}$, we can see that if $P_{1}$ and $P_{2}$ belong to $\mathcal{P}$, then $\xi P_{1}+(1-\xi) P_{2}$ also belongs to $\mathcal{P}$, for any $\xi$ satisfying $0 \leq \xi \leq 1$. We therefore have $\operatorname{co}(\mathcal{P})=\mathcal{P}$. Then $(7)$ gives

$\min _{P \in \operatorname{co}(P)} E_{P} U^{L U}=(1-\delta) E_{C} U^{L U}+\delta \min _{P \in \mathcal{R}(S)} E_{P} U^{L U}$. Substituting this expression into (8), we get (9) .
} 
reason is that uncertainty on the mean of $P^{*}$ tends to make $\sigma_{\gamma}^{2}$ greater than the variance $\sigma^{* 2}$ of $P^{*}$. More CB transparency, which improves the precision of the estimate of the mean of $P^{*}$, reduces this gap and, therefore, decreases $\sigma_{\gamma}^{2}$.

Second, more CB transparency also reduces Knightian uncertainty by reducing $\delta$. This occurs because more numerous or precise data, which are the consequences of more transparency, make any posterior distribution less dependent on the prior held on the underlying parameters of $P^{*}$. In our framework, this tends to reduce $\delta$ because, as indicated in section 2.2 .1 above, $\delta$ is an index measuring this dependence on priors ${ }^{14}$.

We can also note that more "political risk" which creates larger fluctuations of the CB's objectives, and which therefore takes the form of a larger variance $\sigma^{* 2}$ of $P^{*}$, implies a larger variance $\sigma_{\gamma}^{2}$ of $C$.

Therefore, we will do two kinds of comparative statics exercises. The first concerns the variance $\sigma_{\gamma}^{2}$ of the central distribution $C$. The analysis of the effect of $\sigma_{\gamma}^{2}$ generalizes the analysis done (under a Bayesian approach) in the literature by Sorensen (1991) and Grüner (2002), to the case where there is some "ambiguity".

The second concerns the amount of Knightian uncertainty linked to a change in parameter $\delta$. From (10), if we are not in the Bayesian case $\theta=0$ of no aversion to the imprecision of information, more Knightian uncertainty, due to a larger value of $\delta$, implies a greater value of $\alpha$. (In the Bayesian case $\theta=0$ it has no effect).

As an illustration, we will also compare the two opposite cases of complete ignorance and of complete knowledge of $P^{*}$, respectively. In the case of complete ignorance, which could be the consequence of no CB transparency, we have some value $\sigma_{\gamma}^{2}$, which satisfies $\sigma_{\gamma}^{2}>\sigma^{* 2}$. And we have $\delta=1$ because there is no confidence at all on the central distribution. The reason is that lack of knowledge implies that there is no confidence on the central prior on the underlying parameters of $P^{*}$, and it also implies that there are no data which could make the posterior distributions differ from the priors. The opposite case corresponds to the case where a large number of observations or very precise data allow the statistician to estimate $P^{*}$ with (almost) perfect precision. In this case we would have, at the limit, $\mathcal{P}=\left\{P^{*}\right\}$, which corresponds to $\sigma_{\gamma}^{2}=\sigma^{* 2}$ and $\delta=0$ (which implies no Knightian uncertainty). Thus, in the case of complete ignorance we would have $\sigma_{\gamma}^{2}>\sigma^{* 2}$ and $\alpha=\theta$; while in the case of complete knowledge we would have $\sigma_{\gamma}^{2}=\sigma^{* 2}$, and $\alpha=0$.

\footnotetext{
${ }^{14}$ One might also consider that CB transparency could affect the set $S$ which is known to contain the support of $P^{*}$, and therefore could also change parameter $\mu$. However, to simplify the analysis, we will take $S$, and therefore $\mu$, as given.
} 


\section{Effects on the nominal wage.}

\subsection{Nominal wage}

The nominal wage chosen by the LU under uncertainty is the value $\widehat{w}$ of $w$ which maximizes $\Omega^{L U}$ given by $(9)$, where $\mathcal{R}(S)$ is the set of all probability distributions with support in $S=\left[\gamma_{0}-\mu, \gamma_{0}+\mu\right]$.

As a first step, let $\bar{w}(\gamma)$ be the nominal wage that would be chosen by the LU under certainty, when $\gamma$ is known. Maximizing $U^{L U}$ given by (6) gives

$$
\bar{w}(\gamma)=\frac{1}{A a^{2} \gamma}
$$

Then, the nominal wage $\widehat{w}$ chosen by the LU under uncertainty is given by the following proposition (see Appendix 1 for the proof):

Proposition 1 Consider the threshold value $\alpha^{*}$, which satisfies $0 \leq \alpha^{*} \leq 1$, and is given by

$$
\alpha^{*}=\frac{\sigma_{\gamma}^{2}}{\mu\left(\gamma_{0}-\mu\right)+\sigma_{\gamma}^{2}}
$$

In the case $\alpha<\alpha^{*}$, we have

$$
\widehat{w}=\frac{1}{A a^{2}} \frac{\gamma_{0}-\alpha \mu}{\alpha\left(\gamma_{0}-\mu\right)^{2}+(1-\alpha)\left(\gamma_{0}^{2}+\sigma_{\gamma}^{2}\right)}
$$

We have $\widehat{w}<\bar{w}\left(\gamma_{0}\right)$, which means that the nominal wage is lower than under certainty (for $\gamma=\gamma_{0}$ ).

In the case $\alpha \geq \alpha^{*}$, we have $\widehat{w}=\bar{w}\left(\gamma_{0}\right)$. The nominal wage is the same as under certainty $\left(\text { for } \gamma=\gamma_{0}\right)^{15}$.

\subsection{Change in the variance of the central distribution}

In the case $\alpha<\alpha^{*}$, from (13), we get $\frac{\partial \widehat{w}}{\partial \sigma_{\gamma}^{2}}<0$. A larger value of $\sigma_{\gamma}^{2}$ reduces the nominal wage. This was the kind of result found in the literature. Here, we show that this result still holds under the present non-Bayesian approach, as long as "ambiguity" $\alpha$ is not too large (i.e. as long as we are not too far from the Bayesian case $\alpha=0$ ).

\footnotetext{
${ }^{15}$ It is not possible for $\widehat{w}$ to be greater than $\bar{w}\left(\gamma_{0}\right)$. The reason is that, as long as we have $w<\bar{w}\left(\gamma_{0}\right)$, the worst case occurs when $\gamma$ is equal to $\gamma_{0}-\mu$; but, in the case $w>\bar{w}\left(\gamma_{0}\right)$, the worst case changes and becomes $\gamma=\gamma_{0}+\mu$ (see Appendix 2). The discontinuity in the worst cases at $\bar{w}\left(\gamma_{0}\right)$ allows $\bar{w}\left(\gamma_{0}\right)$ to be the solution whenever we have $\alpha \geq \alpha^{*}$.
} 
However, from Proposition 1, when the ambiguity parameter is large enough $\left(\alpha>\alpha^{*}\right)$, there is no effect on the nominal wage of a marginal change in $\sigma_{\gamma}^{2}$. The nominal wage always stays at the level under certainty $\bar{w}\left(\gamma_{0}\right)$, whatever $\sigma_{\gamma}^{2}$ is. In this case, the decrease in the nominal wage, which was found in the literature, does not exist anymore.

\subsection{Change in Knightian uncertainty}

Now consider a change in parameter $\delta$, which can be considered as representing a change in Knightian uncertainty. From (10), if we have $\theta \neq 0$, this implies a change in $\alpha$ of the same sign (nothing is changed in the Bayesian case obtained for $\theta=0$ ).

In the case $\alpha<\alpha^{*}$, from (13), we get $\frac{\partial \widehat{w}}{\partial \alpha}=\frac{1}{A a^{2}} \frac{\left(\gamma_{0}-\mu\right)\left(\sigma_{\gamma}^{2}+\gamma_{0} \mu\right)}{D^{2}}$, where we have $D \equiv \alpha\left(\gamma_{0}-\mu\right)^{2}+(1-\alpha)\left(\gamma_{0}^{2}+\sigma_{\gamma}^{2}\right)$. This gives $\frac{\partial \widehat{w}}{\partial \alpha}>0$.

Therefore, in the case $\alpha<\alpha^{*}$, more Knightian uncertainty, which leads to a greater value of $\alpha$, increases the nominal wage. This effect has a sign which is opposite to the sign found in the case of a larger variance $\sigma_{\gamma}^{2}$ (and is therefore also opposite to the sign of the effect of a larger variance underlined in the literature).

Although our approach is different, we can note that, in this case, we obtain a result similar to that of Schipper and Winschel (2004), who find that greater Knightian uncertainty is likely to increase the nominal wage ${ }^{16}$.

In the case $\alpha>\alpha^{*}$, we have $\frac{\partial \widehat{w}}{\partial \alpha}=0$. Then, a (marginal) change in Knightian uncertainty has no effect on the nominal wage.

\footnotetext{
${ }^{16}$ Schipper and Winschel (2004) have examined the effect of "Knightian uncertainty" in a similar model of interaction between a LU and a CB. But this uncertainty is modelled as strategic uncertainty in a Nash game, which constitutes a different approach. First, these authors consider an equilibrium where the two players play simultaneously, while, in the literature cited and in the present paper, it is essential for the argument that an equilibrium where the LU plays first, is considered. Second, in Schipper and Winschel (2004), in accordance with the literature on ambiguous games, uncertainty is modelled as Knightian uncertainty on the strategy of the other player, which leads to an equilibrium which generalizes the Nash equilibrium. Here, and in the literature cited, uncertainty is on a parameter of the model.
} 


\section{Welfare effects}

\subsection{Criterion}

In this section, we consider the welfare effect ${ }^{17}$. As in the existing literature ${ }^{18}$, we assume that the random fluctuations of the CB's utility function are not part of society's utility function. The CB's weight $\psi$, in the CB's utility function, is random and fluctuates around society's weight $\psi_{0}$. For simplicity, it is further assumed that society's weight $\psi_{0}$ corresponds to the expected value $\gamma_{0}{ }^{19}$. Therefore, society's utility function $U^{s}$ is given by

$$
U^{s}=-\psi_{0} \pi^{2}-u^{2}
$$

where, from (4), $\psi_{0}$ is given by

$$
\psi_{0}=\frac{\gamma_{0}}{1-\gamma_{0}} a^{2}
$$

Then, from (3), (5), (14) and (15), we get

$$
U^{s}=-a^{2}\left[\frac{\gamma_{0}}{1-\gamma_{0}}(1-\gamma)^{2}+\gamma^{2}\right] w^{2}
$$

In order to take into account the fact that parameter $\gamma$ is uncertain, society uses a criterion similar to (9), which is

$$
\Omega^{s}=(1-\alpha) E_{C} U^{s}+\alpha\left[\min _{P \in \mathcal{R}(S)} E_{P} U^{s}\right]
$$

where, as in (9), $\mathcal{R}(S)$ is the set of all probability distributions with support in $\left[\gamma_{0}-\mu, \gamma_{0}+\mu\right]$. For simplicity, we have assumed that society's behavior under uncertainty is characterized by the same parameters $\left(\gamma_{0}, \sigma_{\gamma}^{2}, \mu, \theta, \delta\right)$ as the LU.

Define $\widehat{\Omega}^{s}$ as the value of $\Omega^{s}$ when the nonimal wage $w$ is equal to the level $\widehat{w}$ chosen by the LU. Then, $\widehat{\Omega}^{s}$ is the criterion used by society to evaluate the available information. We can give a more explicit expression to $\widehat{\Omega}^{s}$. We get (see Appendix 2 for a proof) :

\footnotetext{
${ }^{17}$ Sorensen (1991) calls it the effect on "macroeconomic performance". Grüner (2002) considers the effect on inflation uncertainty only.

${ }^{18}$ This is the assumption of Sorensen (1991). But the same kind of assumption is also often made in the literature on CB transparency (e.g. Faust and Svensson (2001)).

${ }^{19}$ Because of the non linearity of the relationship between $\gamma$ and $\psi$, this assumption is not exactly the same as the assumption that $\psi_{0}$ is the mean of $\psi$. We have chosen the assumption which seems simpler for our analysis.
} 
Proposition 2 The criterion used by society to evaluate the available information is

$$
\widehat{\Omega}^{s}=-a^{2} Q \widehat{w}^{2}
$$

where $Q$ is given by

$$
Q=\gamma_{0}+\frac{\alpha \mu^{2}+(1-\alpha) \sigma_{\gamma}^{2}}{1-\gamma_{0}}
$$

Equation (18) indicates that there are two channels through which uncertainty can affect welfare. The first goes through the nominal wage $\widehat{w}$. As, from Proposition 1 and (19), we have $\widehat{w}>0$ and $Q>0$, then (18) implies $\frac{\partial \widehat{\Omega}^{s}}{\partial \widehat{w}}<0$. Thus, a rise in the nominal wage is harmful, while a decrease is beneficial. The effect on the nominal wage has been studied in the previous section.

The second channel goes through coefficient $Q$. From (18), we have $\frac{\partial \widehat{\Omega}^{s}}{\partial Q}<$ 0 . And, from (19), we have $\frac{\partial Q}{\partial \sigma_{\gamma}^{2}}>0$ (except for $\alpha=1$ where we have $\frac{\partial Q}{\partial \sigma_{\gamma}^{2}}=0$ ); and $\frac{\partial Q}{\partial \alpha}>0$ (except for $\sigma_{\gamma}^{2}=\mu^{2}$, where we have $\frac{\partial Q}{\partial \alpha}=0$ ). Therefore, increased "uncertainty" of the kind we consider, corresponding to a higher $\sigma_{\gamma}^{2}$ or to a higher $\alpha$ (resulting from a higher $\delta$ ), has an unfavorable effect through this channel (except in some special cases where there is no effect). This channel corresponds to the harmful effect that an increase in each kind of "uncertainty" has on welfare, when the nominal wage is given.

\subsection{Change in the variance of the central distribution}

We get the following result (see Appendix 3 for a proof):

Proposition 3 (i) In the case $\alpha<\alpha^{*}, \frac{\partial \widehat{\Omega}^{s}}{\partial \sigma_{\gamma}^{2}}$ has the same sign as $Z$ given by

$$
Z=2 \gamma_{0}-3 \gamma_{0}^{2}+\sigma_{\gamma}^{2}+\left(\mu^{2}-\sigma_{\gamma}^{2}+2 \gamma_{0} \mu\right) \alpha
$$

Therefore, a larger variance $\sigma_{\gamma}^{2}$ is beneficial when we have $Z>0$, and is harmful when we have $Z<0$ (with no effect on welfare when $Z=0$ ).

(ii) In the case $\alpha>\alpha^{*}$, we have $\frac{\partial \widehat{\Omega}^{s}}{\partial \sigma_{\gamma}^{2}}<0$ if $\alpha \neq 1$, and $\frac{\partial \widehat{\Omega}^{s}}{\partial \sigma_{\gamma}^{2}}=0$ if $\alpha=1$. Therefore, in this case, when the variance $\sigma_{\gamma}^{2}$ gets larger, welfare is reduced (except in the maxmin case $\alpha=1$, where it is unchanged ${ }^{20}$.

\footnotetext{
${ }^{20}$ In the case $\alpha=\alpha^{*}$, (i) applies when we have $d \sigma_{\gamma}^{2}<0$, while (ii) applies when we have $d \sigma_{\gamma}^{2}>0$.
} 
In the case $\alpha<\alpha^{*}$, as we have underlined in the previous section, a larger value of $\sigma_{\gamma}^{2}$ lowers the nominal wage. This beneficial effect can counterbalance the unfavorable effect going through a higher value of $Q$. This why, depending on the values of the parameters, welfare may be either improved or worsened by a larger variance $\sigma_{\gamma}^{2}$.

The result that a higher variance of the central probability distribution may be beneficial corresponds to the result underlined by Sorensen (1991) in the Bayesian case. Such a possibility is now seen to also hold in the nonBayesian case $\alpha \neq 0$, and we have given the conditions under which welfare is improved by an increase in $\sigma_{\gamma}^{2}$. (These are that both inequalities $\alpha<\alpha^{*}$ and $Z>0$ are satisfied $)^{21}$.

In the case $\alpha>\alpha^{*}$, according to Proposition 1 , the nominal wage is not changed. Therefore, there remains only the second channel, going through $Q$, which is in favor of a reduction in the variance $\sigma_{\gamma}^{2}$ (except in the case $\alpha=1$, where we have $\frac{\partial Q}{\partial \sigma_{\gamma}^{2}}=0$ ) . This is why, in this case, welfare is reduced by an increase in $\sigma_{\gamma}^{2}$. Thus, the result obtained in the literature that a higher variance might be beneficial, does not hold anymore when the ambiguity parameter $\alpha$ (which can be taken as an index of the distance from the Bayesian case) becomes larger than $\alpha^{*}$.

\footnotetext{
${ }^{21}$ It may be instructive to compare the results we obtain in the special case $\alpha=0$, which is the Bayesian case, to what is obtained in the literature under the Bayesian approach. According to our results, in the Bayesian case $\alpha=0$, welfare may either improve or deteriorate, depending on the values of the parameters. From (20), in the case $\alpha=0$, the inequality $Z>0$ becomes equivalent to $\gamma_{0}\left(2-3 \gamma_{0}\right)+\sigma_{\gamma}^{2}>0$. Therefore, when we have $\gamma_{0}<\frac{2}{3}$, we always have $Z>0$, which means that a larger variance always improves welfare. But, in the case $\gamma_{0}>\frac{2}{3}$, we have the opposite result when $\sigma_{\gamma}^{2}$ is not too large, i.e. when we have $\sigma_{\gamma}^{2}<\gamma_{0}\left(3 \gamma_{0}-2\right)$ : then, a larger variance is harmful. However, this ambiguous effect on welfare that we find in the Bayesian case $\alpha=0$, is actually not the same as the ambiguous result of Sorensen (1991), who also found that increased uncertainty may either improve or worsen welfare. In Sorensen (1991), the ambiguity of the result was entirely due to the presence of shocks in the unemployment equation. And, in the absence of shocks, the analysis of Sorensen (1991) actually implies that greater uncertainty always increases welfare (See equation (14) p. 379 in Sorensen (1991)). But, in the present analysis, we do not have such shocks and, nonetheless, we do have an ambiguous effect on welfare.

This discrepancy between our results and those of Sorensen (1991) can be explained by the fact that Sorensen (1991) made the simplifying assumption $\psi_{0}=a=1$, which, from (4), implies $\gamma_{0}=\frac{1}{2}$. But, in this case, the inequality $\gamma_{0}<\frac{2}{3}$ is satisfied. Then, as we have just pointed out, we are in the case where we always have $Z>0$ and where, consequently, an increase in $\sigma_{\gamma}^{2}$ always improves welfare. Therefore, by not restricting the analysis to the special case $\gamma_{0}=\frac{1}{2}$ considered in Sorensen (1991), we find an ambiguous effect on welfare in the Bayesian case $\alpha=0$, even in the absence of shocks.
} 


\subsection{Change in Knightian uncertainty}

From (10), in the Bayesian case $\theta=0$, there is no effect of a change in Knightian uncertainty due to a change in $\delta$. But, when we have $\theta \neq 0$, a change in $\delta$ implies a change in $\alpha$ of the same sign. Then, the two channels previously considered never go into opposite directions. Thus, consider an increase in $\alpha$, which corresponds to a higher amount of Knightian uncertainty. According to the analysis of Section 3, this implies a higher (or unchanged) nominal wage, which has an unfavorable (or null) effect on welfare. In addition, as we have underlined, the second channel, through coefficient $Q$, is also unfavorable, or at most null in some special cases. As a consequence, welfare is in general strictly reduced. It is unchanged only in the special case where both the nominal wage $\widehat{w}$ and coefficient $Q$ are unchanged. According to Proposition 1 and (19), this occurs when we have both $\alpha>\alpha^{*}$ and $\sigma_{\gamma}=\mu$ (this last condition is satisfied when $C$ is concentrated on the two extreme values $\gamma_{0}-\mu$ and $\gamma_{0}+\mu$ ). Therefore, we get

Proposition 4 A greater amount of Knightian uncertainty corresponding to a higher value of $\delta$, never decreases welfare. In general, it strictly reduces welfare. It leaves welfare unchanged only in some special cases (the Bayesian case $\theta=0$; and, when we have $\theta \neq 0$, in the case where both $\alpha>\alpha^{*}$ and $\sigma_{\gamma}=\mu$ hold).

Therefore, the point made in the literature under the Bayesian approach, which is that greater "uncertainty" may improve welfare, does not hold when we consider Knightian uncertainty. In general, greater Knightian uncertainty (strictly) reduces welfare.

\subsection{Implications}

As we have indicated in section 2.2.3 above, more CB transparency has two effects. One is to reduce the variance $\sigma_{\gamma}^{2}$ of the central distribution $C$. The other is to reduce Knightian uncertainty, because of a smaller $\delta$. As we have seen, this last effect is favorable to more CB transparency, because a decrease in Knightian uncertainty is never harmful (and is in general beneficial). Therefore, in the case where ambiguity is not too large $\left(\alpha<\alpha^{*}\right)$, the possible unfavorable effect due a lower $\sigma_{\gamma}^{2}$ is dampened, and may even be dominated by the favorable effect due to a smaller amount of Knightian uncertainty. In the case where ambiguity is large enough $\left(\alpha>\alpha^{*}\right)$, both effects contribute to make CB transparency beneficial. Thus, when we are far enough from the Bayesian case, the result obtained in the literature that CB transparency can be harmful never holds. 
In the Bayesian analysis done in the literature, neither the beneficial effect going through reduced Knightian uncertainty, nor the fact that a reduced variance $\sigma_{\gamma}^{2}$ is favorable when ambiguity is large enough, were taken into account. For these two reasons, our non-Bayesian analysis gives results more favorable to CB transparency than those obtained in the literature under the Bayesian approach.

As an illustration, consider what happens when we go from complete ignorance to complete knowledge of the underlying probability distribution $P^{*}$. Such a move may be viewed as an extreme case of the effect of $\mathrm{CB}$ transparency. As indicated in Section 2.2.3 above, this means that we go from $\left(\sigma_{\gamma, 1}^{2}>\sigma^{* 2} ; \alpha_{1}=\theta\right)$ to $\left(\sigma_{\gamma, 2}^{2}=\sigma^{* 2} ; \alpha_{2}=0\right)$.

In the Bayesian case $\theta=0$, the favorable effect going through reduced Knightian uncertainty is absent. But, according to (13), the smaller value of $\sigma_{\gamma}^{2}$ would increase the nominal wage. This has an unfavorable effect on welfare. On the other hand, there is a direct favorable effect on welfare through a smaller value of coefficient $Q$ in (18): $\sigma_{\gamma, 1}^{2}>\sigma_{\gamma, 2}^{2}$ implies $Q_{2}<$ $Q_{1}$. This is why, depending on the parameters, such a move from complete ignorance to complete knowledge of $P^{*}$ can either be beneficial or harmful. A sufficient condition for this change to be harmful is that it is harmful for all marginal changes considered along the path. According to Proposition 3 (with $\alpha=0$ ), this is equivalent to having $\gamma_{0}\left(2-3 \gamma_{0}\right)+\sigma_{\gamma}^{2}>0$ for all $\sigma_{\gamma}^{2}$ such that $\sigma^{* 2} \leq \sigma_{\gamma}^{2} \leq \sigma_{\gamma, 1}^{2}$, which is equivalent to having $\gamma_{0}\left(2-3 \gamma_{0}\right)+\sigma^{* 2}>0$. In the case $\gamma_{0}<\frac{2}{3}$, this condition is always fulfilled. Such a move, which could be due to more CB transparency, would be harmful in this case. This corresponds to the kind of result that was underlined in the literature under the Bayesian approach.

In the non-Bayesian case $\theta>0$, results may become quite different when $\theta$ is large enough. This occurs when the beneficial effect of the elimination of Knightian uncertainty always dominate the potentially harmful effect of a reduced variance of the central distribution. Thus, consider the case $\theta>\alpha_{1}^{*}$ (where $\alpha_{1}^{*}$ is given by (12), where $\sigma_{\gamma}^{2}$ is replaced by $\sigma_{\gamma, 1}^{2}$ ). Then, as we have $\alpha_{1}=\theta>\alpha_{1}^{*}$, Proposition 1implies that, under complete ignorance, the nominal wage $\widehat{w}_{1}$ is equal to the level under certainty $\bar{w}\left(\gamma_{0}\right)$. On the other hand, under complete knowledge, $\widehat{w}_{2}$ is smaller than $\bar{w}\left(\gamma_{0}\right)$ (at least as long as we have $\left.\sigma^{*}>0\right)^{22}$. This decrease in the nominal wage improves welfare. This

\footnotetext{
${ }^{22}$ Under complete knowledge of $P^{*}$, as we have $\alpha_{2}=0$, we are in the case $\alpha_{2}<\alpha_{2}^{*}$ (where $\alpha_{2}^{*}$ is given by (12) and $\sigma_{\gamma}^{2}=\sigma^{*}$ ) if we have $\sigma^{*}>0$.

Note that if we had $\sigma^{*}=0$, and therefore $\alpha_{2}^{*}=0$, the nominal wage $\widehat{w}_{2}$ would be equal to $\bar{w}\left(\gamma_{0}\right)$, and therefore be the same under complete knowledge as under complete ignorance. But there would still remain the favorable direct effect on welfare going through a smaller value of coefficient $Q$. Therefore, even in the case $\sigma^{*}=0$, such an increase in
} 
effect is reinforced by the direct effect going through coefficient $Q$ in (18): as both $\sigma_{\gamma}^{2}$ and $\alpha$ are smaller, we have $Q_{2}<Q_{1}$. Consequently, the overall effect on welfare is always favorable. A sufficiently large aversion to the imprecision of information $\theta$ can therefore make such a move from complete ignorance to complete knowledge of the underlying probability distribution $P^{*}$, always beneficial. The result, obtained in the Bayesian case, that such a move can be harmful, does not hold in this case.

Finally, as indicated in section 2.2.3 above, more political risk implies a larger variance $\sigma_{\gamma}^{2}$ of the central distribution $C$. Therefore, the effect of political risk directly follows from the previous analysis of the effect of $\sigma_{\gamma}^{2}$. Thus, when we are far enough from the Bayesian case because ambiguity is large enough $\left(\alpha>\alpha^{*}\right)$, more political risk is never beneficial (and in general is harmful). It is only when ambiguity is not too large $\left(\alpha<\alpha^{*}\right)$, i.e. when we are not too far from the Bayesian case, that we obtain the result underlined in the literature that more political risk could be beneficial.

\section{Conclusion}

The literature on central bank transparency mainly uses an expected utility Bayesian approach to uncertainty. Here, we have underlined that the results obtained may be very different when we depart from this Bayesian approach and use a non-Bayesian approach to uncertainty, which contains the Bayesian approach as a special case, but which, more generally, introduces an aversion to ambiguity and Knightian uncertainty.

We have considered a specific argument which appears in the literature. This argument shows that more uncertainty on central bank (CB) preferences could be beneficial because, in a game between the $\mathrm{CB}$ and a monopoly labor union (LU), this would lead the LU to lower the nominal wage. Such a result has been interpreted as an argument showing that political uncertainty, which creates fluctuations of $\mathrm{CB}$ objectives, may be beneficial; and also as an argument against CB transparency, because more CB transparency would tend to reduce the uncertainty that the private sector has on $\mathrm{CB}$ preferences.

We have tried to extend and revaluate this argument (obtained under the standard Bayesian expected utility approach in the literature) by using a non-Bayesian framework.

In our framework, as in the literature, political factors create fluctuations of CB preferences. These fluctuations follow some probability distribution which is only imperfectly known to the private sector. Under the non-Bayesian approach we have considered, the information available to the

CB transparency would always be beneficial. 
LU has two parts. First, there is a "central probability distribution" which reflects two things: the fluctuations of the CB's preferences, on the one hand; and the estimate of this distribution under some central prior on the parameters underlying this distribution, on the other hand. More political risk, which leads to more fluctuations of CB preferences, increases the variance of this central distribution. And more CB transparency, which increases the accuracy of the estimates, reduces this variance.

Second, in addition to this central distribution, the information available to the private sector also takes the form of some Knightian uncertainty around this central probability distribution. Such an uncertainty results from the lack of confidence that the private sector has on its priors when it tries to estimate the probability distribution which governs the fluctuations of the CB's preferences. More CB transparency, which increases the amount and quality of data, reduces this Knightian uncertainty by diminishing the importance of priors relatively to data in the resulting estimates.

We have considered the effects of the corresponding two parameters which are affected by CB transparency: the variance of the central distribution, on the one hand; and a parameter which an index of the amount of Knightian uncertainty, on the other hand.

When we consider the effect of a change in the variance of the central distribution, the level of "ambiguity" has been shown to play a crucial role. "Ambiguity" is a mix of two parameters of the model: the "aversion to the imprecision of information" of the decision maker, on the one hand; and a parameter which is an index of the amount of Knightian uncertainty, on the other hand. This ambiguity parameter can be considered as an index measuring the distance from the Bayesian case. When ambiguity is lower than some threshold value, then a greater variance of the central distribution lowers the nominal wage and, consequently, can be beneficial for some values of the parameters. As a consequence, greater fluctuations of the CB's preferences, due to more political risk, can be beneficial; and, as more CB transparency decreases the variance of the central distribution, this also gives an argument against CB transparency. This result can be seen as a generalization of the result obtained in the literature in the Bayesian case of no ambiguity, to the non-Bayesian case, where ambiguity is not zero, but still not too large.

However, when ambiguity becomes larger than the threshold value, a greater variance of the central distribution does not affect the nominal wage (which stays equal to the nominal wage under certainty), and, consequently, can never be beneficial (and is in general harmful). This means that the result obtained in the literature under the Bayesian approach, does not hold when ambiguity is large enough: in this case, more political risk is in general harmful; and the effect of CB transparency which goes through a reduced 
variance of the central distribution, becomes favorable.

As long as ambiguity is not equal to zero, i.e. as soon as we depart from the Bayesian case, more Knightian uncertainty is never beneficial. In general, it is harmful. There are two reasons for that. First, the nominal wage is never lowered (and is even raised when ambiguity is not too large), and, second, for a given nominal wage, increased Knightian uncertainty tends to decrease welfare.

This favorable effect on welfare of reduced Knightian uncertainty is an other argument in favor of CB transparency which was not taken into account in the literature. When ambiguity is not too large, the favorable effect going through reduced Knightian uncertainty dampens, or may even dominate, the possible unfavorable effect due to a reduced variance of the central distribution that more CB transparency may imply. And, when ambiguity is large enough, as the two effects go into the same direction, the favorable effect of reduced Knightian uncertainty further contributes to make CB transparency beneficial.

As an illustration, we have considered the extreme case where a change in CB transparency would imply a move from complete ignorance to complete knowledge of the underlying probability distribution followed by the CB's preferences. In the Bayesian case, such a move can be harmful. However, when the aversion to the imprecision of information is large enough, then, because of the favorable effect due to the elimination of Knightian uncertainty, such a move has been shown to be always beneficial.

Therefore, in the presence of ambiguity and of Knightian uncertainty, the specific argument that we have considered (which, in the literature, was developed under a Bayesian approach) may become an argument for transparency rather than against transparency.

\section{Appendices}

\section{Proof of Proposition 1}

Consider the term $\min _{P \in \mathcal{R}(S)} E_{P} U^{L U}$ in (9). The function $U^{L U}(\gamma, w)$ given by (6) is a quadratic concave function of $\gamma$. As a consequence, in (9), the probability distribution with support $\left[\gamma_{0}-\mu, \gamma_{0}+\mu\right]$ which minimizes $E_{P} U^{L U}$ is obtained either for the probability distribution which gives $\gamma=\gamma_{0}-\mu$ with probability one, or for the probability distribution which gives $\gamma=\gamma_{0}+\mu$ with probability one. We get $\gamma=\gamma_{0}-\mu$ as a solution when we have $U^{L U}\left(\gamma_{0}-\mu, w\right) \leq U^{L U}\left(\gamma_{0}+\mu, w\right)$. Using (6) and (11), this can be written $2 \mu A a^{2} \gamma_{0} w\left[w-\bar{w}\left(\gamma_{0}\right)\right] \leq 0$. Therefore, as we have $\bar{w}\left(\gamma_{0}\right)>0$, the value 
$\gamma=\gamma_{0}-\mu$ is a solution in the case $0 \leq w \leq \bar{w}\left(\gamma_{0}\right)$, while $\gamma=\gamma_{0}+\mu$ is a solution in the cases $w \leq 0$ and $w \geq \bar{w}\left(\gamma_{0}\right)$ (and there is indifference between $\gamma_{0}-\mu$ and $\gamma_{0}+\mu$ when $w=0$ or $\left.w=\bar{w}\left(\gamma_{0}\right)\right)$.

Consider the function $\widetilde{\Omega}^{L U}(\gamma, w)$ defined by

$$
\widetilde{\Omega}^{L U}(\gamma, w) \equiv \alpha U^{L U}(\gamma, w)+(1-\alpha) E_{C} U^{L U}(\gamma, w)
$$

Using (6), we can see that $\widetilde{\Omega}^{L U}(\gamma, w)$ is a quadratic concave function of $w$ which is maximized for $\widetilde{w}(\gamma)$ given by

$$
\widetilde{w}(\gamma)=\frac{1}{A a^{2}} \frac{\alpha \gamma+(1-\alpha) \gamma_{0}}{\alpha \gamma^{2}+(1-\alpha)\left(\gamma_{0}^{2}+\sigma_{\gamma}^{2}\right)}
$$

On the interval $]-\infty, 0]$, as we just have shown, we have $\min _{P \in \mathcal{R}(S)} E_{P} U^{L U}=$ $U^{L U}\left(\gamma_{0}+\mu\right)$ and therefore, from (9), we have $\Omega^{L U}=\widetilde{\Omega}^{L U}\left(\gamma_{0}+\mu, w\right)$. As a consequence, on the interval ]- $\infty, 0]$, the value of $w$ which maximizes $\Omega^{L U}$ is equal to $\min \left[\widetilde{w}\left(\gamma_{0}+\mu\right), 0\right]$. As we have $\widetilde{w}\left(\gamma_{0}+\mu\right)>0$, this is equal to 0 . On the interval $\left[\bar{w}\left(\gamma_{0}\right),+\infty\right.$ [ we also have $\min _{P \in \mathcal{R}(S)} E_{P} U^{L U}=U^{L U}\left(\gamma_{0}+\mu\right)$, and therefore the value of $w$ which maximizes $\Omega^{L U}$ on this interval is equal to $\max \left[\widetilde{w}\left(\gamma_{0}+\mu\right), \bar{w}\left(\gamma_{0}\right)\right]$. Using (11) and (22) we can see that we always have $\widetilde{w}\left(\gamma_{0}+\mu\right)<\bar{w}\left(\gamma_{0}\right)$. Therefore, on the interval $\left[\bar{w}\left(\gamma_{0}\right),+\infty[\right.$, the optimal value of $w$ is $\bar{w}\left(\gamma_{0}\right)$. These results imply that 0 is (strictly) better than any value of $w$ which belongs to $]-\infty, 0\left[\right.$, and that $\bar{w}\left(\gamma_{0}\right)$ is (strictly) better than any value of $w$ which belongs to $] \bar{w}\left(\gamma_{0}\right),+\infty[$. As a consequence, the value $\widehat{w}$ which maximizes $\Omega^{L U}$, given by (9), has to belong to the interval $\left[0, \bar{w}\left(\gamma_{0}\right)\right]$. But we have shown that on $\left[0, \bar{w}\left(\gamma_{0}\right)\right]$ we have $\min _{P \in \mathcal{R}(S)} E_{P} U^{L U}=U^{L U}\left(\gamma_{0}-\mu\right)$, and therefore $\Omega^{L U}=\widetilde{\Omega}^{L U}\left(\gamma_{0}-\mu, w\right)$. As we have $\widetilde{w}\left(\gamma_{0}-\mu\right)>0$, this implies $\widehat{w}=\min \left[\widetilde{w}\left(\gamma_{0}-\mu\right), \bar{w}\left(\gamma_{0}\right)\right]$. Using (11) and (22), we get that $\min \left[\widetilde{w}\left(\gamma_{0}-\mu\right), \bar{w}\left(\gamma_{0}\right)\right]$ is equal to $\widetilde{w}\left(\gamma_{0}-\mu\right)$ in the case $\alpha<\alpha^{*}$, and to $\bar{w}\left(\gamma_{0}\right)$ in the case $\alpha>\alpha^{*}, \widetilde{w}\left(\gamma_{0}-\mu\right)$ and $\bar{w}\left(\gamma_{0}\right)$ being equal in the case $\alpha=\alpha^{*}$. The two cases of Proposition 1 directly follow. The inequality $\widehat{w}<\bar{w}\left(\gamma_{0}\right)$ comes from (11), (13) and the inequality $\mu<\gamma_{0}$. QED

\section{Proof of Proposition 2}

First, consider the term $\min _{P \in \mathcal{R}(S)} E_{P} U^{S}$ in (17). From (16), this is equivalent to $\max _{P \in \mathcal{R}(S)} E_{P}\left[\frac{\gamma_{0}}{1-\gamma_{0}}(1-\gamma)^{2}+\gamma^{2}\right]$. As the term into brackets is a quadratic convex function of $\gamma$, the maximum can be attained only on the set of the two extreme distributions where we have $\gamma=\gamma_{0}-\mu$ with probability one, or $\gamma=\gamma_{0}+\mu$ with probability one. It can be seen that $\gamma=\gamma_{0}-\mu$ and 
$\gamma=\gamma_{0}+\mu$ actually always give the same value to $\frac{\gamma_{0}}{1-\gamma_{0}}(1-\gamma)^{2}+\gamma^{2}$. Thus, replacing $\gamma$ by $\gamma_{0}-\mu$, or equivalently by $\gamma_{0}+\mu$, in (16) we get

$$
\min _{P \in \mathcal{R}(S)} E_{P} U^{s}=-a^{2}\left(\gamma_{0}+\frac{\mu^{2}}{1-\gamma_{0}}\right) w^{2}
$$

Second, from (16), we obtain $E_{C} U^{s}=-a^{2}\left(\gamma_{0}+\frac{\sigma_{\gamma}^{2}}{1-\gamma_{0}}\right) w^{2}$. Substituting this expression and (23) into (17), and replacing $w$ by $\widehat{w}$, we get (18) and (19). QED

\section{Proof of Proposition 3}

From (18), we have

$$
\frac{\partial \widehat{\Omega}^{s}}{\partial \sigma_{\gamma}^{2}}=-a^{2} \widehat{w}\left(2 Q \frac{\partial \widehat{w}}{\partial \sigma_{\gamma}^{2}}+\widehat{w} \frac{\partial Q}{\partial \sigma_{\gamma}^{2}}\right)
$$

In the case $\alpha<\alpha^{*}$, using (13), (19) and (24), we obtain after calculus $\frac{\partial \widehat{\Omega}^{s}}{\partial \sigma_{\gamma}^{2}}=G(1-\alpha) Z$, where we have $G \equiv \frac{\widehat{w}}{A\left(\alpha\left(\gamma_{0}-\mu\right)^{2}+(1-\alpha)\left(\gamma_{0}^{2}+\sigma_{\gamma}^{2}\right)\right)^{2}} \frac{\gamma_{0}-\alpha \mu}{1-\gamma_{0}}>0$, and where $Z$ is given by (20). As we are in the case $\alpha<\alpha^{*}$, and as we have $\alpha^{*} \leq 1$, we necessarily have $\alpha<1$, which implies that $\frac{\partial \widehat{\Omega}^{s}}{\partial \sigma_{\gamma}^{2}}$ has the sign of $Z$.

In the case $\alpha>\alpha^{*}$, according to proposition 1 , we have $\frac{\partial \widehat{w}}{\partial \sigma_{\gamma}^{2}}=0$. From (24), this implies that $\frac{\partial \widehat{\Omega}^{s}}{\partial \sigma_{\gamma}^{2}}$ has the opposite sign of $\frac{\partial Q}{\partial \sigma_{\gamma}^{2}}$. But from (19), we have $\frac{\partial Q}{\partial \sigma_{\gamma}^{2}}>0$ if $\alpha \neq 1$, and $\frac{\partial Q}{\partial \sigma_{\gamma}^{2}}=0$ if $\alpha=1$. QED

\section{References}

[1] Berger, J. and L.M. Berliner (1986), "Robust Bayes and Empirical Bayes Analysis with $\varepsilon$-Contaminated Priors", The Annals of Statistics, Vol. 14, No. 2, 461-486.

[2] Blinder, A.S., M. Ehrmann, M. Fratzscher, J. De Haan and D.-J. Jansen (2008), "Central Bank Communication and Monetary Policy: A Survey of Theory and Evidence", Journal of Economic Literature, 46:4, 910-945.

[3] Cukierman, A. and F. Lippi (1999), "Central Bank Independence, Centralization of Wage Bargaining, Inflation and Unemployment: Theory and Some Evidence", European Economic Review, 43, 1395-1434.

[4] Dai, M. and M. Sidiropoulos (2008), Central Bank's Conservativeness and Transparency, Research in Economics, 62, 179-187. 
[5] Eichberger, J. and D. Kelsey (1999), "E-Capacities and the Ellsberg Paradox", Theory and Decision, 46, 107-140

[6] Ellsberg, D. (1961),"Risk, Ambiguity, and the Savage Axioms", Quarterly Journal of Economics, 75,.643-669.

[7] Epstein, L.G. and T. Wang (1994), "Intertemporal Asset Pricing under Knightian Uncertainty", Econometrica, 62 (3), March.

[8] Faust, J. and L.E.O. Svensson (2001), "Transparency and Credibility: Monetary Policy with Unobserved Goals", International Economic Review, Vol. 42, No. 2, May, 369-397.

[9] Gajdos, T., Tallon, J.-M. and J.-C. Vergnaud (2004), "Decision Making with Imprecise Probabilistic Information", Journal of Mathematical Economics, 40, 647-681.

[10] Gilboa, I. and D. Schmeidler ((1989), "Maximin Expected Utility with Non-Unique Prior", Journal Mathematical Economics,18, 141-153.

[11] Grüner, H.P. (2002), "How Much Should Central Banks Talk? A New Argument", Economics Letters, 77, 195-198.

[12] Knight, F.H. (1921), Risk, Uncertainty and Profit, Boston: Houghton Mifflin.

[13] Mukerji, S. and J.-M. Tallon (2004), "An overview of economic applications of David Schmeidlers's models of decision making under uncertainty" in I.Gilboa, (ed), Uncertainty in Economic Theory: A collection of essays in honor of David Schmeidler's 65th birthday, Routledge Publishers.

[14] Schipper, B.C. and E. Winschel (2004), "Knightian Intransparency of Central Bank and Labor Union", May.

[15] Schmeidler, D. (1989), "Subjective Probability and Expected Utility without Additivity", Econometrica, Vol.57, No. 3, May, 571-587.

[16] Sorensen, J.R. (1991), "Political Uncertainty and Macroeconomic Performance", Economics Letters, 37, 377-381.

[17] Van der Cruijsen, C. and S. Eijffinger (2007), "The Economic Impact of Central Bank Transparency: A Survey", Center for Economic Research Discussion Paper, Tilburg University, January. 